All letters must be typed with double spacing and signed by all authors.

No letter should be more than 400 words.

For letters on scientific subjects we normally reserve our correspondence columns for those relating to issues discussed recently (within six weeks) in the BMF.

We do not routinely acknowledge letters. Please send a stamped addressed envelope if you would like an acknowledgment.

Because we receive many more letters than we can publish we may shorten those we do print, particularly when we receive several on the same subject.

\section{Mortality in meningococcal disease}

SIR, - Meningococcal disease remains one of the most serious acute infections presenting to general practitioners, and sometimes considerable diagnostic skill is required to differentiate it from a host of self limiting viral infections. The peak incidence is in the winter, particularly from December to March.' Notifications and laboratory isolations of meningococci have shown an upward trend for seven of the past eight years; the Meningococcal Reference Laboratory received strains from 1500 cases in $1990 .^{1}$ In 1990,169 deaths were attributable to meningococcal disease in England and Wales, of which $64 \%$ were in children aged under 15; mortality has probably changed little in recent years.

We have analysed retrospectively the survival of patients with meningococcal disease in our health districts. In three of the four districts mortality was considerably reduced when patients with suspected meningococcal disease were treated by their general practitioner with parenteral benzylpenicillin before admission to hospital. The effect of early penicillin treatment was greatest among patients with a petechial or purpuric rash; these patients have a higher mortality.

These data will be submitted for publication. In the interim we urge all general practitioners to carry benzylpenicillin in their emergency bags and to consider the possibility of meningococcal disease. Not infrequently the initial rash has little if any petechial or purpuric component ${ }^{2}$; repeated examination may be necessary. A suspected diagnosis of meningococcal disease constitutes a medical emergency.

\section{Dogs that bite}

SIR,-There are some difficulties in interpreting the data presented in figure 1 of $\mathrm{P} \mathrm{C}$ Shewell and J D Nancarrow's paper on dog bites ${ }^{1}$; this figure gives the number of dog bites in children and adults by breed of dog. To assess how dangerous these breeds are and their relative tendency to bite we need to know not only the frequency with which they bite but how this relates to the numbers of these breeds in the general canine population and the amount of time the dogs spend around people.

"Medium sized mongrels," for example, must be the most common variety of dog on our streets and in our homes and yet they accounted for only nine of the bites to adults and one to children. The authors of the paper were surprised that "Dobermann pinschers and Rottweilers were responsible for only $7 \%$ of bites." It is not possible to make any sense of this statement until we know
A single injection of benzylpenicillin should be given immediately intramuscularly or (preferably) intravenously. An appropriate dose is $1200 \mathrm{mg}$ ( 2 megaunits) for adults and children over 10 years, $600 \mathrm{mg}$ for children aged $1-10$ years, and $300 \mathrm{mg}$ for infants. Most patients with a history of "penicillin allergy" can safely be given a dose of penicillin as above, ${ }^{3}$ but if a risk of anaphylaxis is thought to exist chloramphenicol (an immediate dose of $12.5 \mathrm{mg} / \mathrm{kg}$ for children or $1 \mathrm{~g}$ for adults), which must be given intravenously, is a suitable alternative. Both antibiotic powders are stable for at least two years in an emergency bag. After being given the antibiotic the patient should be transported to hospital as rapidly as possible. Prompt treatment of meningococcal disease may be life saving.

Public Health Laboratory,

Gloucestershire Royal Hospital,

Gloucester GL1 3NN

KEITH CARTWRIGH'T

Department of Public Health Medicine, Memorial Hospital,

Darlington DL36HX

Communicable Disease Control

Plymouth Health Authority,

Plymouth PL6 5XP

Public Health Laboratory

Royal United Hospital (North),

Bath BA1 3NG

1 Jones DM, Kaczmarski EB. Meningococcal infections in England and Wales: report of the Meningococcal Reference Laboratory for 1990. Communicable Disease Report 1991;1 R76-8.

2 Baxter P, Priestley B. Meningococcal rash. Lancet 1988;i:1166-7. 3 Surtees SJ, Stockton MG, Gietzen TW. Allergy to penicillin fable or fact? BMF 1991;302:1051-2.

how many of these relatively rare breeds there are and how much time they spend with people. The little Jack Russell seems to get a particularly raw deal from this presentation as it is said to account for $13 \%$ of bites, yet we do not know whether this is principally because it is a popular breed.

Dogs presumably spend more time in the presence of children than of adults, although clearly this varies from breed to breed. Certainly, dogs spend more time in the home and around their owners than they do elsewhere, so it should come as no surprise that most adults are bitten at home by their own dogs.

These problems related to the presentation and interpretation of data are known to epidemiologists as problems of the "floating numerator"-that is, a numerator floating without its denominator, in the absence of which it cannot be interpreted. In this context the problem conjures up images of medium sized mongrels, Dobermann pinschers, and Jack Russells floating in search of their population denominators, presumably snapping and snarling as they do so.

RHYS WILLIAMS

Department of Community Medicine,

University of Cambridge,

Addenbrooke's Hospital,

Addenbrooke's Hos

Trevelyan College,

University of Durham

Durham

1 Shewell PC, Nancarrow JD. Dogs that bite. BMF 1991;303: 1512-3. (14 December.)

SIR, - In their report of a study of patients referred for primary treatment of dog bites P C Shewell and J D Nancarrow point out that their patients were a selected group but note that dog bites are common.

We previously reported results of surveys of dog bites in Thanet and Salisbury and concluded that nearly a quarter of a million dog bites are treated annually in British accident and emergency departments. ${ }^{2}{ }^{3}$ In the study from Salisbury, out of 225 people with dog bite injuries identified in one year, only one was referred to a plastic surgeon. A crude extrapolation of the figures from Shewell and Nancarrow's study suggests that about four million of the population of West Midlands are referred to plastic surgeons with dog bites each year.

A very small proportion of dog bites are seen by plastic surgeons, and care should be taken in suggesting that the types of dog that bit the patients seen by plastic surgeons, or the circumstances in which the bites occurred, are typical for all dog bites treated in the United Kingdom; a previous study, for instance, found that half the dogs concerned were strays. ${ }^{5}$ It would be wrong to consider control of dogs to be largely a domestic issue.

SIMON N VOSS

Department of Public Health and HUGH F THOMAS

Epidemiology,

Odstock Hospital,

Odstock Hospital

1 Shewell PC, Nancarrow JD. Dogs that bite. BMF 1991;303 $1512-3$.

2 Thomas HF, Banks J. A survey of dog bites in Thanet. IR Soc Health 1990;110:173.

3 Thomas HF, Voss SN. A survey of dog bites in Salisbury. fR Soc Health 1991;111:224-5.

4 Department of Health. Public health common data set. London: DoH, 1991.

5 Talbot D. Medical hazards from dogs. BMF 1985;291:280.

SIR, - I was interested in P C Shewell and J D Nancarrow's paper on dog bites' as they listed a review of which I was a coauthor ${ }^{2}$; I agree that we gave little attention to the aetiology or prevention 